\title{
Relationship between smartphone use and musculoskeletal symptoms in adolescents
}

\author{
P.C. Santos ${ }^{1,2}$, R. Oliveira ${ }^{1}$, C. Mesquita ${ }^{3, *}$, J. Mota $^{2}$, R. Azevedo ${ }^{1}$, S. Lopes ${ }^{1}$ \\ ${ }^{1}$ School of Allied Health \\ Technologies/Polytechnic Institute of Porto, Department of Physiotherapy, Porto, Portugal \\ ${ }^{2}$ Research Centre in Physical Activity, Health and Leisure, Faculty of Sport, University of Porto, \\ Porto, Portugal \\ ${ }^{3}$ Health School - Politecnico do Porto, Department of Physiotherapy, Porto, Portugal
}

Purpose: We are currently facing a society of adolescents who are increasingly dependent on technology, in particular the smartphone, and this phenomenon can even lead to limiting situations in which the person's physical wellbeing is called into question. Intensive use of the smartphone may contribute to a decrease in physical activity and generate musculoskeletal symptoms (MMS).

$\operatorname{Aim}(\mathbf{s}):$ To verify the existence of a relationship between the use of the smartphone and: (1) MMS; (2) vigorous, moderate and sedentary physical activity.

Methods: An observational, analytical, cross-sectional study was conducted on a sample of 834 adolescents from five schools in the Viseu, Vila Real and Porto regions. Data collection was performed through online questionnaires through the Qualtrics program to make the sociodemographic characterization of the sample and determination of behavioral habits related to health, as well as the use of new technologies. Musculoskeletal symptoms were evaluated through the Portuguese version of the Nordic musculoskeletal questionnaire. (NMQ) and physical activity through the International Questionnaire of Physical Activity (IPAQ).

Results: The adolescents who used the smartphone for the most time referred MMS in the cervical $(P<$ $0.001)$, tho-racic $(P=0.017)$, lumbar $(P<0.001)$, shoulders $(P<0.001)$, wrists/hands $(P=0.003)$ and knees $(P=0.013)$. Adolescents who practice more vigorous physical activity $(P=0.023)$ use less smartphone, and those who have more time in sedentary physical activity $(P=0.008)$ use it more.

Conclusion(s): Adolescents who spend more time on smartphones refer more MMS. The use of the smartphone is associated with a more sedentary lifestyle, unlike the adolescents who practice vigorous physical activity that give less use to him.

Implications: The high time of use of smartphones suggests the importance of health promotion campaigns in this population. 